\title{
Study on particle movement characteristics in sandblast cylinder based on PIV technology
}

\author{
Zhiquan Hui ${ }^{*}$, Feng Wu², Haojie Duan², Bo Yang', Jinmei Lin', Lian Wang', Si Huang³ \\ ${ }^{1}$ Guangzhou Special Pressure Equipment Inspection and Research Institute, Guangzhou 510663, China \\ ${ }^{2}$ School of Chemical Engineering, Northwest University, Xian 710069, China \\ ${ }^{3}$ School of Mechanical and Automotive Engineering, South China University of Technology, Guangzhou 510641,China \\ *Corresponding author:275530236@qq.com
}

\begin{abstract}
PIV technology was used to investigate the influence of the particles' relevant parameters on the instantaneous movement characteristics in the sandblast cylinder under the circumstance of different particle sizes, different section heights and different stacking conditions. As the diameter increased, particles had a greater velocity and energy when approaching the wall, which would cause a serious abrasion. The influence of test selection factors on the particles' radial velocity of particles was greater than that on axial velocity. The radial velocity and axial velocity on the surface of the cylindrical section were all reduced to a lower level when the particles approach the tank wall. When $r>0.3 \mathrm{R}$, the particle velocity maintained at a higher level, but then decreased slowly when $\mathrm{r}$ $>0.7 \mathrm{R}$. Therefore, the abrasion of the conical section of the sandblast cylinder when $\mathrm{r}>0.3 \mathrm{R}$ should be paid more attention to.
\end{abstract}

Keywords: PIV technology, Sandblast cylinder, Particles, Movement characteristics.

\section{INTRODUCTION}

Sandblasting machine is a common mechanical cleaning equipment for pneumatic conveying of sand particles, which has a very important application in the fields such as, oil pipe, inner wall rust removal, ship section surface treatment, etc ${ }^{1}$. Sandblasting machine is mainly composed of sandblast cylinder and spray gun. Its working principle is to spray abrasive grains from sandblast cylinder to working surface with high-speed spout formed by compressed air, so as to change its mechanical properties and achieve the purpose of processing ${ }^{2}$. When sandblasting, the abrasives in the sandblast cylinder flow in a complex way, which continuously affects and abrades the inner wall of the sandblast cylinder. As time goes by, the wall becomes thinner and thinner. When the wall thickness is reduced to a certain extent, it won't be able to withstand the high pressure from the internal air flow, and the sandblast cylinder will break or even explode, thus causing serious security risks. Solid particle erosion is a common problem existing in oil and gas engineering. The wear of a conveyor system caused by solid particle erosion will increase the equipment's maintenance and repair costs and even lead to serious production accidents $^{3}$. In addition, it will also bring a huge economic loss to the actual production ${ }^{4}$.

The abrasion of sandblast cylinder belongs to a solid phase abrasion. According to the relevant abrasion theory, this process is the impact and cutting process of abrasive particles on tank wall. The amount of abrasion is proportional to the kinetic energy $\left(\mathrm{mv}^{2} / 2\right)$ and the velocity of particles ${ }^{5}$. The impact angle between particles and tank wall, the size of particles, the density of particles, the hardness of tank wall materials and the abrasion greatly impact the abrasion of the $\operatorname{tank}^{6-8}$. The angular particles will produce a more serious wear than the spherical smooth particles under the same conditions. Meanwhile, the particle velocity is divided into two types, including the velocity component perpendicular to the wall surface of the sandblast cylinder (radial U) and the velocity component parallel to the wall surface of the tank (axial V). The radial velocity mainly produces a deformation and wear on the surface of the tank wall, while the axial velocity mainly produces a cutting wear on the surface of the tank wall'. Among the influencing factors, the particles' kinetic energy (or velocity) has a direct positive correlation with the wear, which has been widely recognized. Therefore, it is important to explore the influence law of the change in limited factors on the particle velocity under the circumstance of a certain material and particle characteristics of sandblast cylinder. In addition, the influence law can guide the structural design and the optimal operation of sandblast cylinder .

PIV(Particle Image Velocimetry) technology can track and record the instantaneous velocity of numerous particles in a certain section of the flow field simultaneously, so as to analyze the law of particle motion characteristics. PIV technology has been widely used in the following fields, including fluid flow ${ }^{\mathbf{1 0}}$, chemical engineering Research ${ }^{11}$, nuclear reaction engineering ${ }^{12}$, building-environment ${ }^{13}$ as well as Ocean Engineering ${ }^{14}$. Now, its application has been expanded to 3D PTV and PSV(Particle Streak Velocimetry) ${ }^{\mathbf{1 5}}$, which increases the application scope while improving the accuracy ${ }^{16}$. Wu et al. used PIV technology to study the $0.72 \mathrm{~mm}$ $\sim 1.41 \mathrm{~mm}$ glass sand in spouted bed ${ }^{17}$, with a bright prospect. Based on the structural characteristics of the cylinder, a transparent sandblast cylinder structure model was manufactured. The particles coated by $\mathrm{SiO}_{2}$ are selected for PIV experiments to facilitate the high-speed cameras to capture the particle phase motion, and PIV test technology is adopted to observe and analyze the particle velocity vector and its component characteristics under the circumstance of different particle sizes, stacking heights as well as filling states in the sandblast cylinder, and then explore the relevant influence laws. As the wear mainly occurs at the edge of the cylinder section rather than the center, the movement characteristics of the particles focus on the change trend from the center of the section to the edge of the interface. 


\section{INTRODUCTION TO TEST MODEL, EQUIPMENT AND METHOD}

\section{Test model}

The test model is a glass column conical sandblast cylinder with the specification of $45^{\circ}$ inverted cone angle at the bottom, and the inner diameter of the gas inlet nozzle is $25 \mathrm{~mm}$. The side of the bottom of sandblast cylinder is provided with two spray holes with an inner diameter of $25 \mathrm{~mm}$, and the top is connected to a sealing cap by a flange, as shown in Fig. 1 .

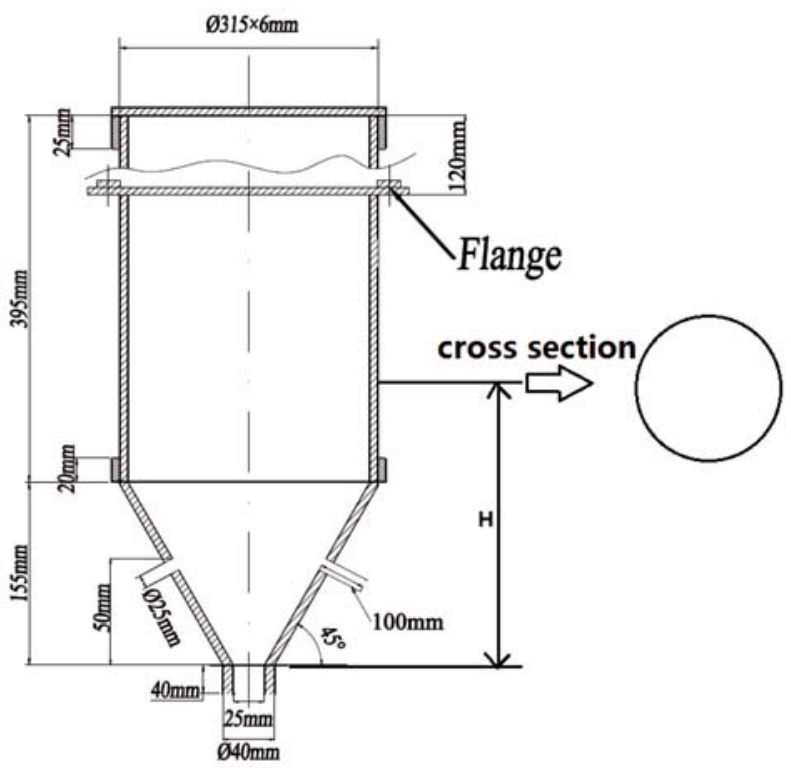

Figure 1. Structural drawing of sandblast cylinder for test

\section{Test equipment}

In this test, the laser chip light source of PIV system is the Mini-YAG double pulse laser manufactured by NewWave company, and its maximum energy is $200 \mathrm{MJ}$, maximum pulse frequency is $21 \mathrm{~Hz}$, and emission wavelength is $532 \mathrm{~nm}$ green light. The CCD camera adopts the FlowSense 4M MKII camera of Dantec company, with a full frame pixel resolution of $2048 \times 2048$ and a maximum frame rate of 21 pairs $\cdot \mathrm{s}^{-1}$, as shown in Fig. 2.

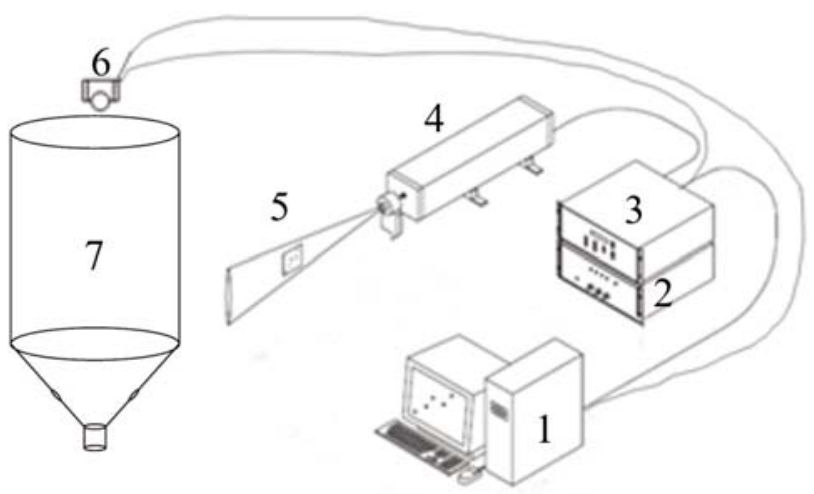

Figure 2. Structural drawing of test equipment for test: 1 . Analysis system; 2. Synchronizer; 3. Image processing; 4. Laser; 5. Light emitting surface; 6. Camera; 7. Sandblast cylinder.

\section{Test method}

In the experiment, an air compressor was used to blow the air in. The gas inlet flow was $400 \mathrm{~L} \cdot \mathrm{min}^{-1}$, and the height of the inlet horizontal plane was zero. According to the performance of the test equipment, the default height of the acquisition cross-section $\mathrm{H}$ (that is, the height of the measured cross-section from the inlet horizontal plane) was $240 \mathrm{~mm}$. The frosted materials with a high reflectivity were selected for the experiments. The diameter of the particles was $1.4 \mathrm{~mm}$ (16\#), $0.85 \mathrm{~mm}(24 \#), 0.71 \mathrm{~mm}(30 \#)$ and $0.6 \mathrm{~mm}$ (36\#), respectively. The particle density (@) was 2200 $\mathrm{kg} / \mathrm{m}^{3}$. The morphology characteristics of particles with different sizes under SEM are shown in Fig. 3.1 to Fig. 3.4. It can be seen that the particles were irregular and polygonal, which is more prone to have a wear on the surface of the tank wall.

Table 1. Gas-solid medium properties

\begin{tabular}{|c|c|c|c|}
\hline Medium & Density $\left[\mathrm{kg} / \mathrm{m}^{3}\right]$ & $\begin{array}{c}\text { Diameter } \\
{[\mathrm{mm}]}\end{array}$ & Viscosity $\left[\mathrm{m}^{2} / \mathrm{s}\right]$ \\
\hline Air & 1.29 & - & $14.8 \times 10^{-6}$ \\
\hline Particles & 2200 & $0.6 \sim 1.4$ & - \\
\hline
\end{tabular}

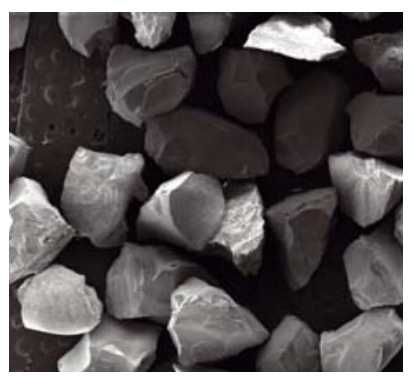

Figure 3.1. Morphology of particles 16

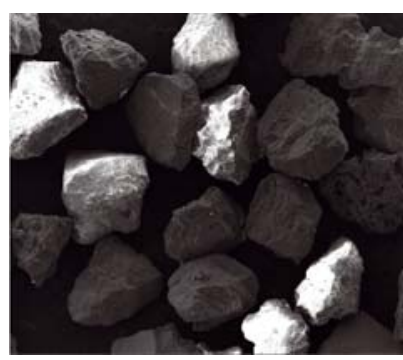

Figure 3.2. Morphology of particles 24

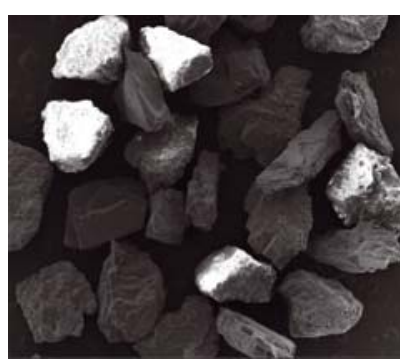

Figure 3.3. Morphology of particles 30

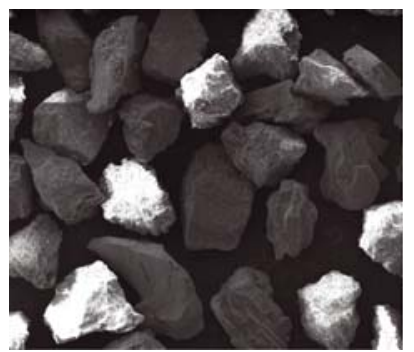

Figure 3.4. Morphology of particles 36 
When the particles in the tank moved to a stable state, PIV was used to capture and record the movement of the particles with a high reflectivity in the tank, and dynamic Studio software was used to process and analyze the measurement results. The radial and axial velocity distribution of moving particles was obtained. Time interval between adjacent sampled pictures is $2000 \mu \mathrm{s}$. The value range of the test section was $200 \mathrm{~mm} \times 200 \mathrm{~mm}$, which was limited by the test-bed conditions of the test equipment, and the change of particle velocity vector in the flow field was analyzed in this section.

As shown in Fig. 4 and Fig. 5, two different paths were selected to characterize the particle velocity trajectory. Figure 4 shows the transverse intercept of velocity vector (line 1: $\mathrm{y}=140 \mathrm{~mm}$, line 2: $\mathrm{y}=100 \mathrm{~mm}$ (centerline), lin 3: $y=60 \mathrm{~mm}$ ), which represents the specific change of particle velocity on the specific track. Figure 5 shows the change of the average velocity of different distances $\left(\mathrm{R}_{1}=0.1 \mathrm{R}, \mathrm{R}_{2}=0.2 \mathrm{R}, \mathrm{R}_{3}=0.4 \mathrm{R}, \mathrm{R}_{4}=0.5 \mathrm{R}, 0.6\right.$ $\left.\mathrm{R}, \mathrm{R}_{5}=0.7 \mathrm{R}, \mathrm{R}_{6}=0.8 \mathrm{R}, \mathrm{R}_{7}=0.87 \mathrm{R}, \mathrm{R}=100 \mathrm{~mm}\right)$ from the central jet axis, which represents the overall change of particle velocity. Considering the particles' peripheral flow, the truncated circle selected is sparse inside and dense outside.

The test flow aims to study the movement characteristics of the particles with different sizes at the same cross-section height, then select the movement characteristics of the particles with the same size at different height cross-section, and finally select the movement characteristics of the particles with the same size under different filling conditions.

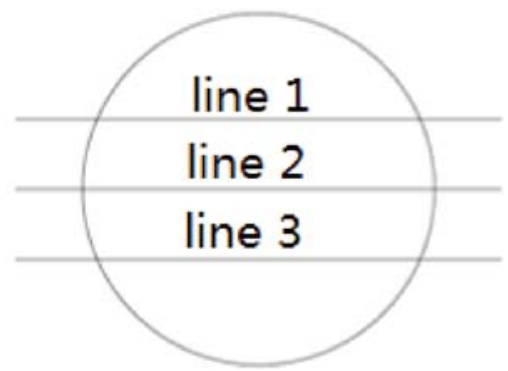

Figure 4. Straight line selection position of sandblast cylinder cross section

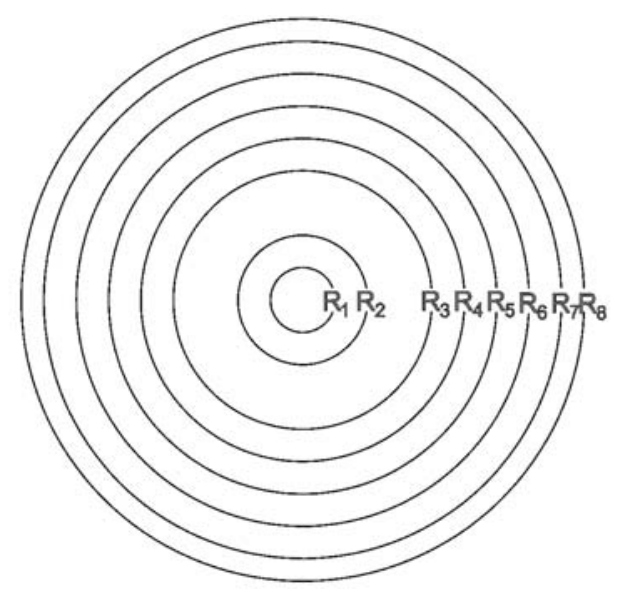

Figure 5. Circular selection position of sandblast cylinder section

\section{TEST RESULTS AND ANALYSIS}

Test results under different particle diameters (H $=240 \mathrm{~mm})$

\section{Velocity(total) vector distribution under different particle diameters}
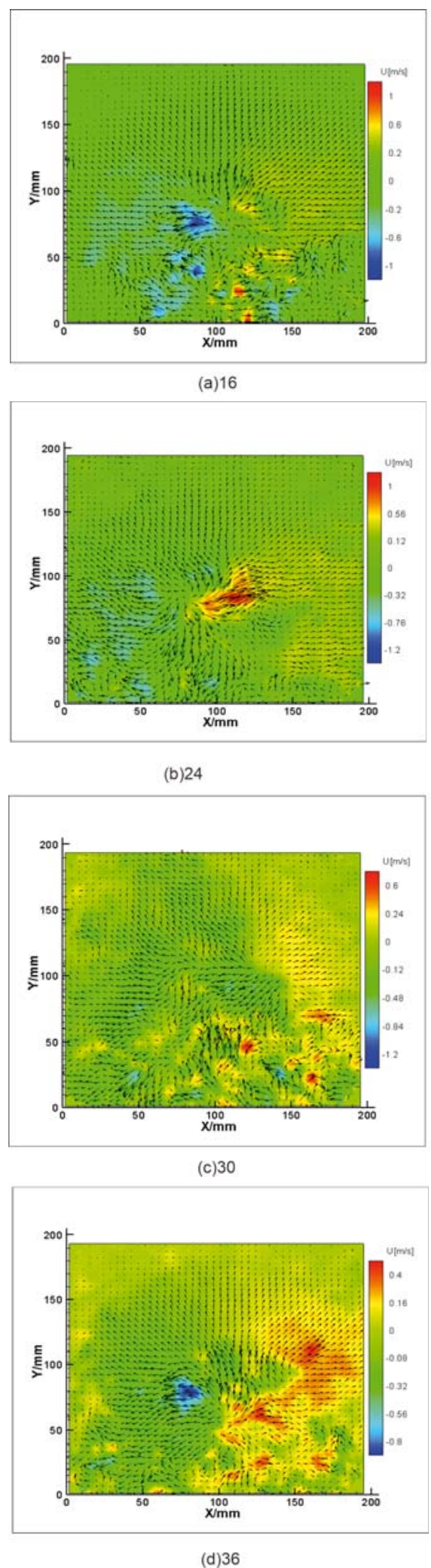

Figure 6. Velocity vector distribution under different particle diameters 
In the sandblast cylinder, the gas is injected directly from the nozzle below, which drives the particles to move into the tank. The two particle outlets are located on the side wall of the cone section, which affects the stable movement of the particles. The vector lines in the figure only represent the movement trend, and the color depth shows the speed. Vortex and disturbance appear in some areas, as shown in the red area of Figure 6. It is observed that most of the unstable areas appear in the lower right corner of the cross section. Therefore, it is speculated that the large air flux on the right side of the cross section results in the local particles' violent movement. However, this parameter is affected by the specific installation operation of sandblast cylinder as well as the structure of the air inlet and outlet. In the practical engineering application, the particles may have a more serious wear in this specific area than in other areas, which needs to be paid more attention to.

From the overall velocity distribution of the inner section of the tank under the four particle diameters in Figure 6, it can be seen that, with the increase of the diameter, the velocity distribution range of the particles gradually reduces, which may be due to the decrease of the particle diameter, the increase of the number of particles and the filling density under the same filling height. Therefore, it not only results in the decrease of the average energy of the final particles, but also reduces the damage to the sandblast cylinder.

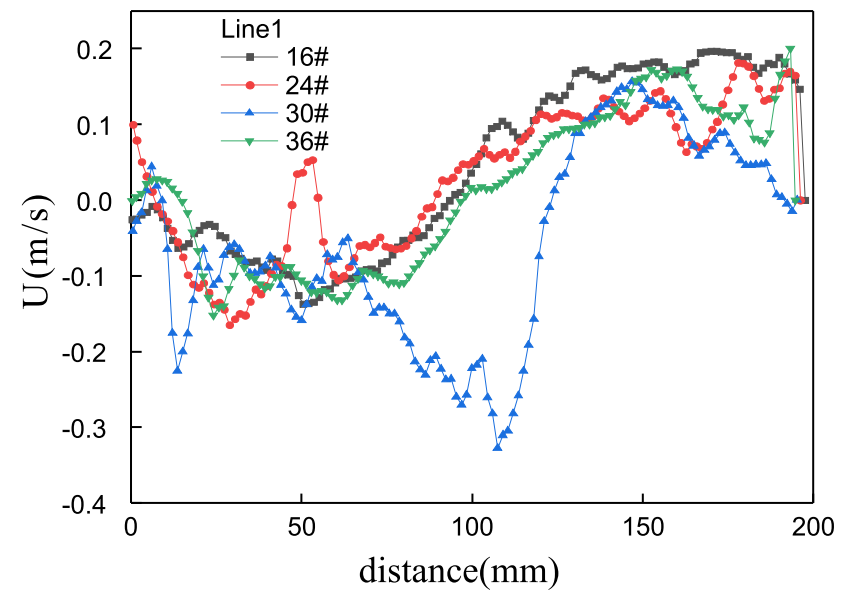

Figure 7.1. Radial velocity distribution of particles with different diameters on line 1

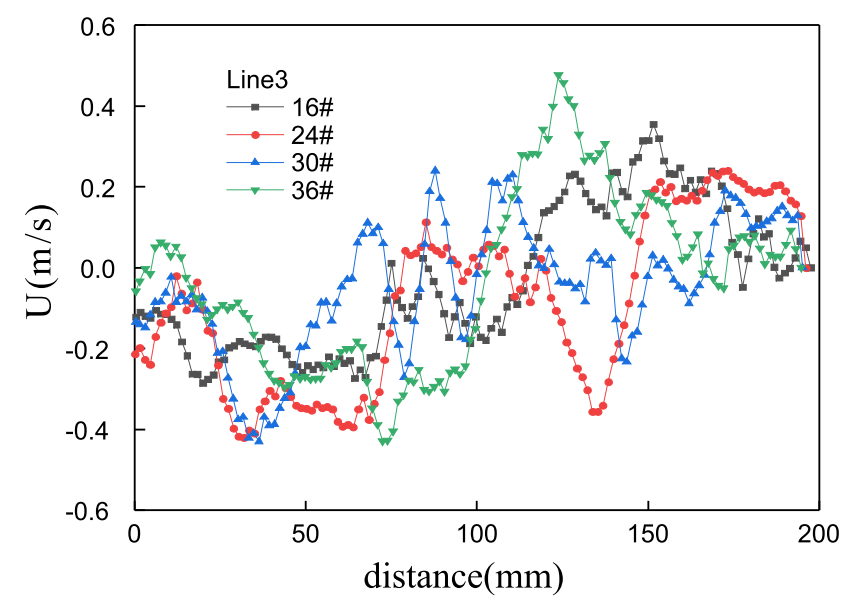

Figure 7.3. Radial velocity distribution of particles with different diameters on line 3

\section{Radial velocity vector $(\mathrm{U})$ distribution under different particle diameters}

As shown in Fig. 7.1 to Fig. 7.3, the radial velocity of particles with different diameters along the standard line presents a fluctuant distribution from the tank center $(100 \mathrm{~mm})$ to the tank walls on both sides. It reaches the velocity peak within $50 \mathrm{~mm}$ from the center and then gradually reduces. The radial velocity at the edge of the section $(200 \mathrm{~mm})$ is around 0 . The velocity distribution along the center line line 2 is larger. Line 1 and line 3 are geometrically located in the opposite direction, but their velocity distribution is different, which reflects the complexity of the flow field in the tank.

According to the radial velocity distribution of particles with different diameters under different circular section tracks shown in Figure 7.4, it can be seen that during the movement of particles with different diameters from the center position to the tank wall, the radial velocity shows a gradual downward trend, and the radial velocity of particles with smaller diameters drops more obviously, which may be because the particles with larger diameters have a greater energy inertia, thus affecting the wall surface more seriously.

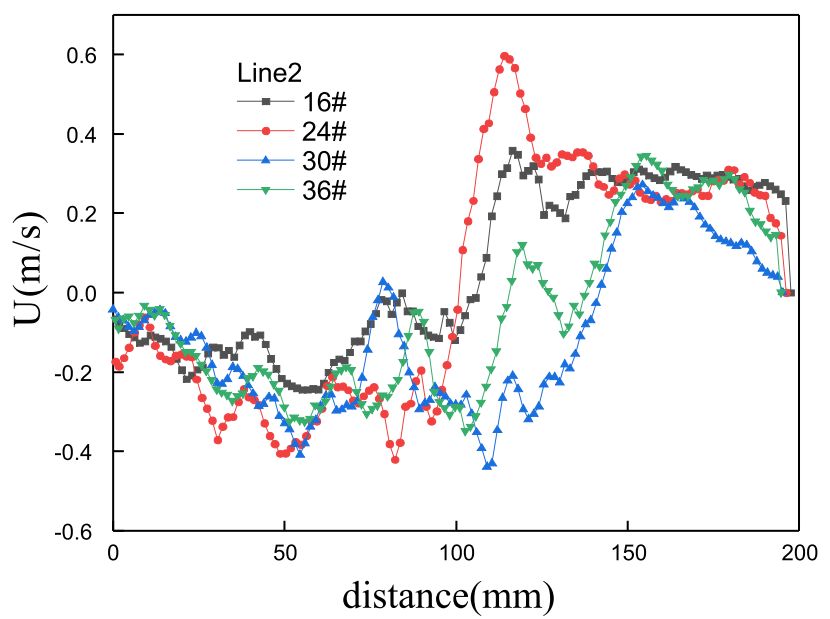

Figure 7.2. Radial velocity distribution of particles with different diameters on line 2

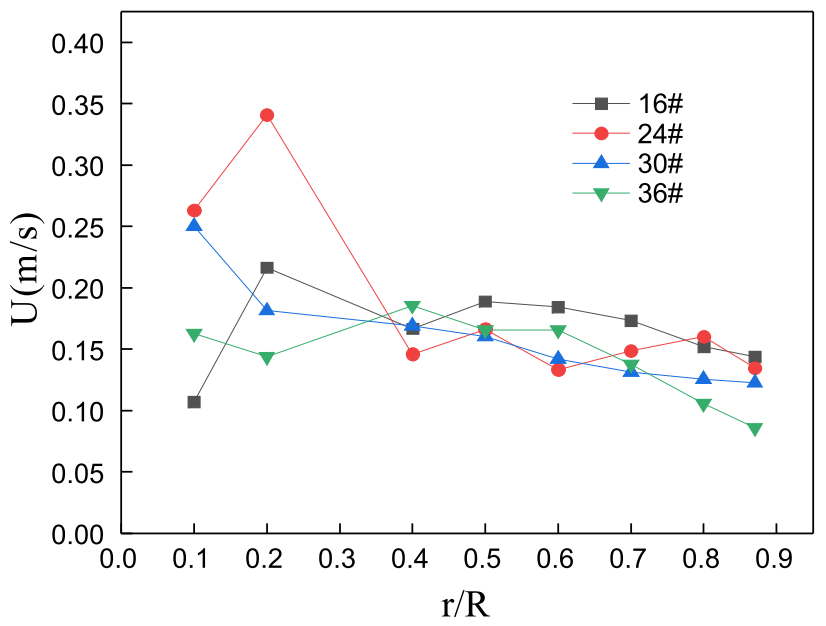

Figure 7.4. Radial velocity distribution of particles with different diameters under different circular section trajectories 
Axial velocity vector (V) distribution under different particle diameters

As shown in Fig. 8.1 to Fig. 8.3, the axial velocity of particles with different diameters along the standard line presents a fluctuant distribution from the tank center to the tank walls on both sides, and it reaches the peak value near the position $100 \mathrm{~mm}$ away from the center, and then gradually reduces. The axial velocity at the edge of the cross section is all around 0 . The axial velocity of particles with different diameters at the edge of line 3 section $(200 \mathrm{~mm})$ shows certain differentiation characteristics; the velocity distribution of particles along the center line line 2 is larger, and line 1 and line 3 are located in the geometric symmetry position, but their velocity distribution is quite different, which reflects the complexity of the flow field in the tank. According to the axial velocity distribution of particles with different diameters under different circular section tracks shown in Fig. 8.4, it can be seen that, during the movement of the particles with different diameters from the center position to the tank wall direction, the overall axial velocity shows a gradual downward trend, and the axial velocity values of particles with different diameters tend to be about $0.9 \mathrm{R}$, which indicates that the particle diameter has a weak impact on the axial velocity of particles at the edge of the section.

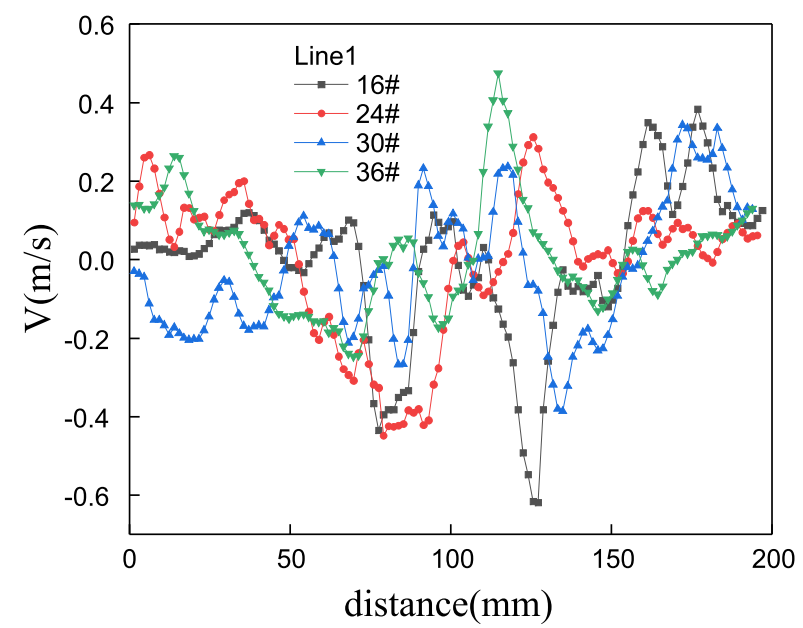

Figure 8.1. Axial velocity distribution of particles with different diameters on line 1

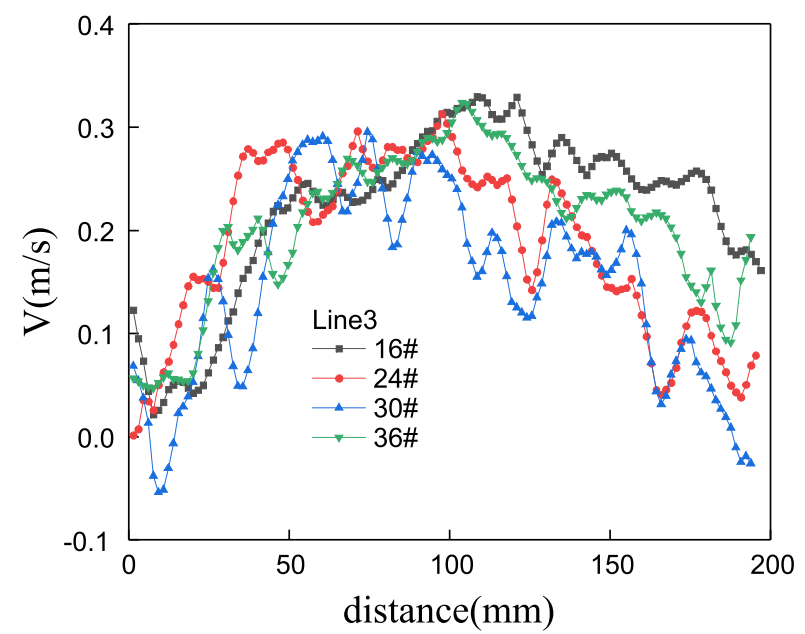

Figure 8.3. Axial velocity distribution of particles with different diameters on line 3
At the same time, as shown in Fig. 8.1 to Fig. 8.3, it can be seen that under the geometric characteristics of the sandblast cylinder with the test model conditions, the radial velocity and axial velocity of the particles with different diameters drop to a lower level at the edge of the section $(\mathrm{H}=240 \mathrm{~mm})$.

Test results under different irradiation cross sections (particle size 16\#)

\section{Velocity vector distribution under different irradiation} cross sections

As shown in Fig. 9.1 to Fig. 9.3, it can be seen that a strong turbulence occurs in some areas due to the uneven distribution of the outlet gas flux of the sandblast cylinder. At the same time, a slight instability can be seen in Fig. 9.3, but its range and change are small, so it is considered that its impact on the wall surface can be ignored compared with other steady-state flows.

Meanwhile, from the color distribution of Figures 9.1 to 9.3 , it can be seen that the lower section presents stronger velocity vector characteristics, which may cause a greater wear on the wall surface of the sandblast cylinder.

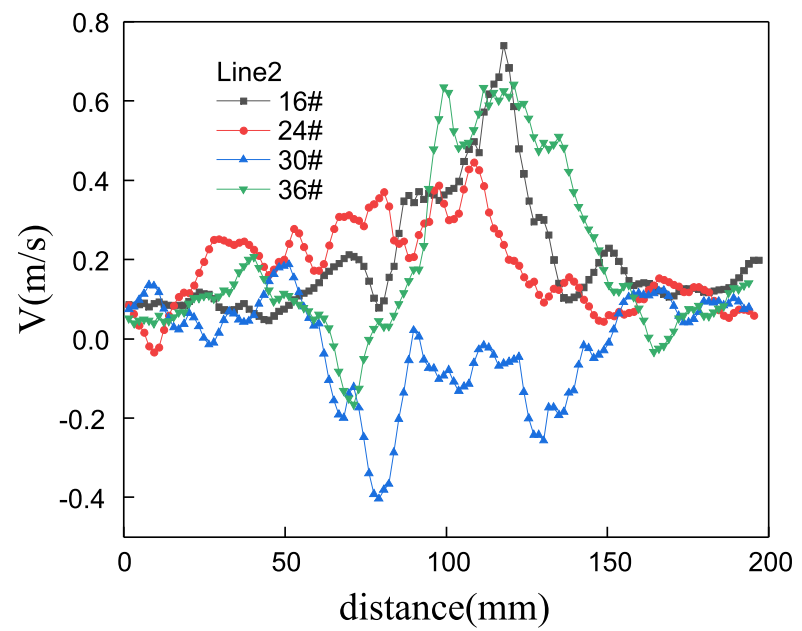

Figure 8.2. Axial velocity distribution of particles with different diameters on line 2

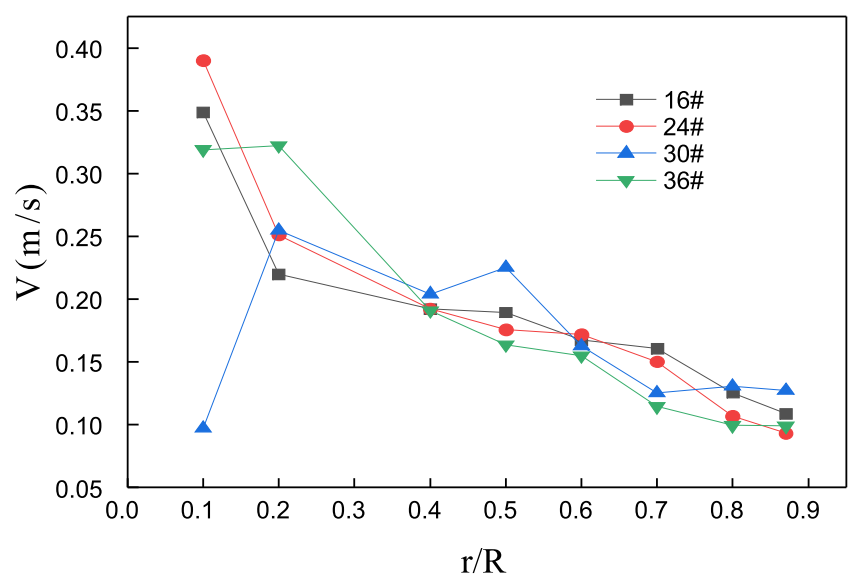

Figure 8.4. Axial velocity distribution of particles with different diameters under different circular section trajectories 

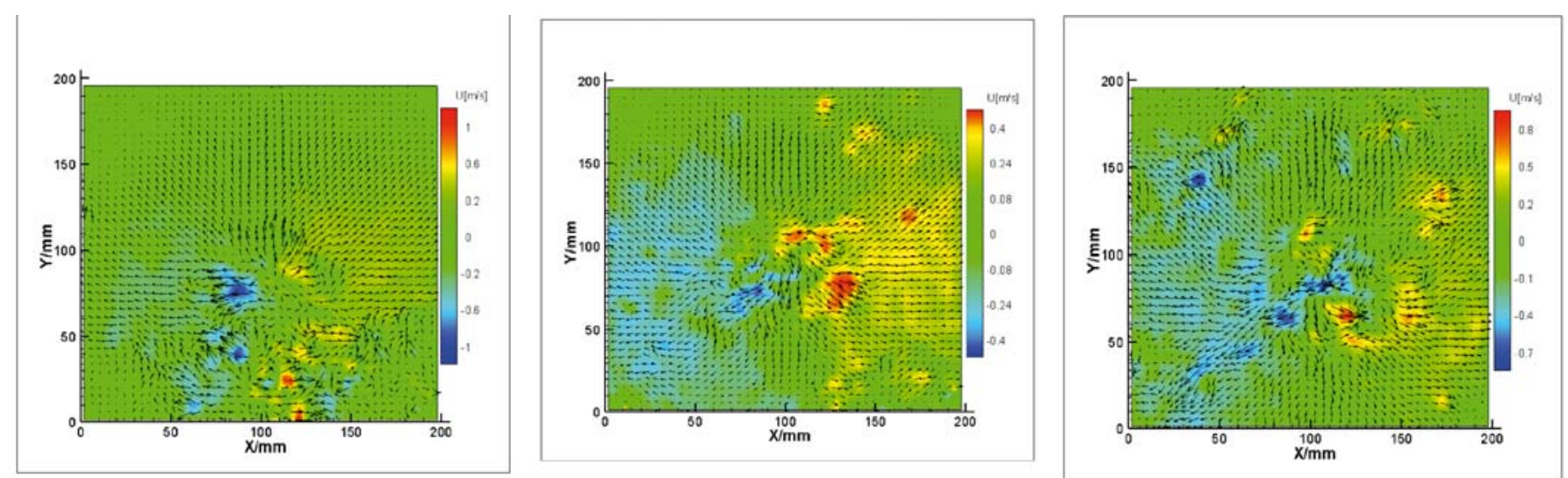

Figure 9.1. Velocity vector distribution under Figure 9.2. Velocity vector distribution under different irradiation cross sections $(\mathrm{H}=240 \mathrm{~mm})$ different irradiation cross sections $(\mathrm{H}=280 \mathrm{~mm})$

Figure 9.3. Velocity vector distribution under different irradiation cross sections $(\mathrm{H}=320 \mathrm{~mm})$

\section{Radial velocity vector (U) distribution under different irradiation cross sections}

As shown in Fig. 10.1 to Fig. 10.3, the radial velocity of 16\# particles in different sections along line 2 and line 3 presents a fluctuant distribution from the center of the tank to the both sides of the tank wall. It reaches the peak velocity near the center and then gradually reduces. The radial velocity at the edge of the section is about 0 . The radial velocity of particles at a section height of $\mathrm{h}=$ $320 \mathrm{~mm}$ presents a larger fluctuation feature. The radial

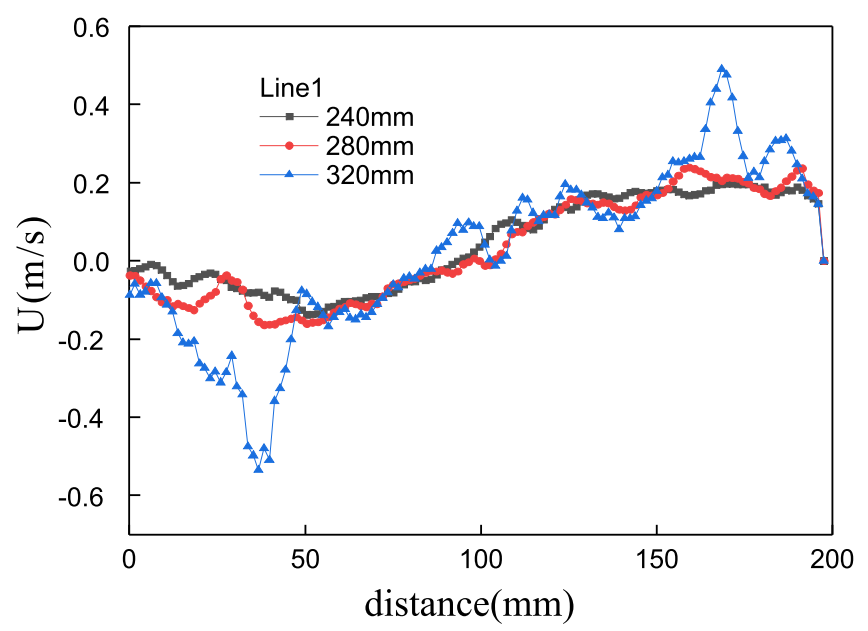

Figure 10.1. Radial velocity distribution of particles under different irradiation cross sections on line 1

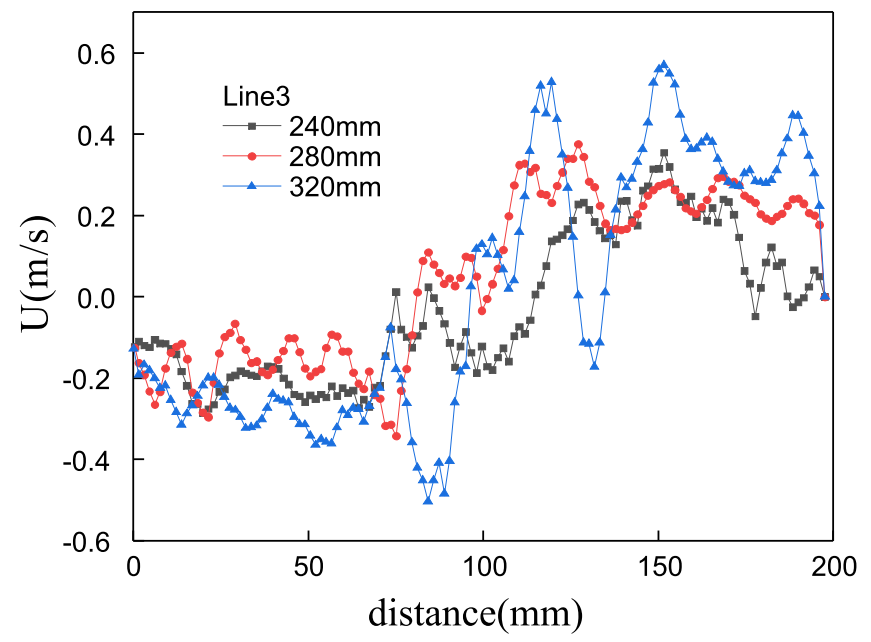

Figure 10.3. Radial velocity distribution of particles under different irradiation cross sections on line 3 velocity of the three sections on line 1 is relatively consistent, and they are all in a low speed state, which may be due to the small influence of air flow on the standard line 1. According to the radial velocity distribution of particles in different sections under different circular section tracks shown in Figure 10.4, it can be seen that the radial velocity shows a gradual downward trend when the particles in different sections move from the center position to the tank wall direction. The decrease in the particles' radial velocity at high section is more obvious,

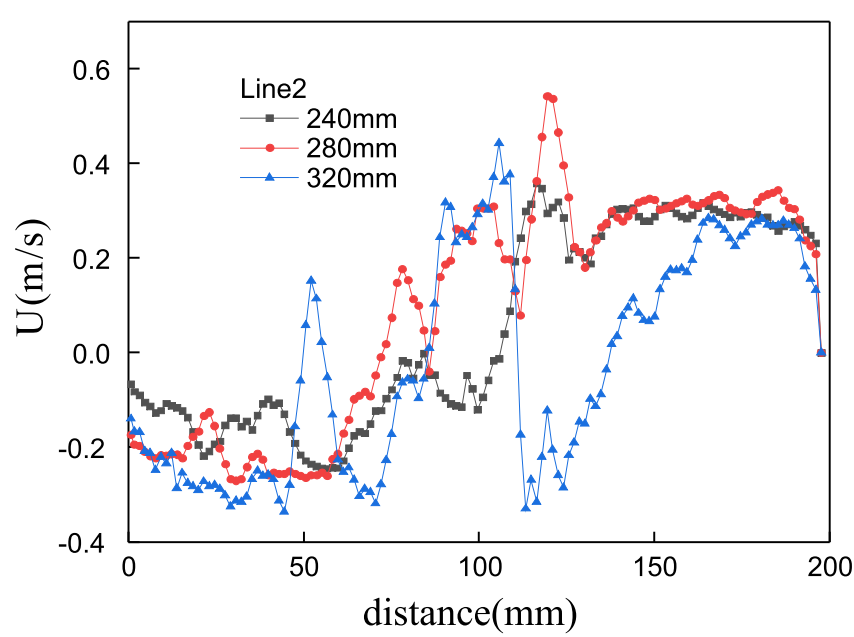

Figure 10.2. Radial velocity distribution of particles under different irradiation cross sections on line 2

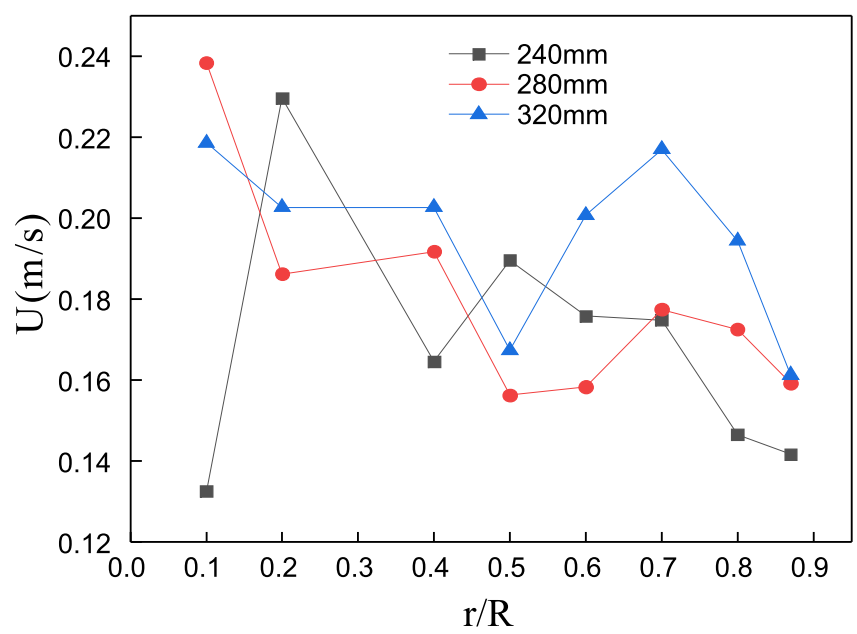

Figure 10.4. Radial velocity distribution of particles under different irradiation cross sections along different circular section trajectories 
while that at low section is more moderate. Therefore, it can be judged that the damage of particles to the tank wall is negatively correlated with the height, and the lower the height is, the damage intensity will be.

\section{Axial velocity vector (V) distribution under different} irradiation cross sections

As shown in Fig. 11.1 to Fig. 11.3, the axial velocity of $16 \#$ particles in different sections along the line presents a fluctuant distribution from the center of the tank to the both sides of the tank wall. It reaches a peak velocity near the center and then gradually reduces. On the center line, namely, line 2, the particles with a lower cross-section present a greater fluctuation characteristic, and on both sides, namely, line 1 and line 3, the particles with a higher cross-section present a greater fluctuation characteristic, but the axial velocity at the edge of the cross section is at a low level. According to the axial velocity distribution of particles in different sections under different circular section tracks shown in Fig. 11.4, it can be seen that the axial velocity shows a gradual downward trend when the particles in different sections move from the center position to the tank wall direction. The axial velocity of particles in different sections approaching to the edge of the section is basically the same downward rate, indicating that the section height

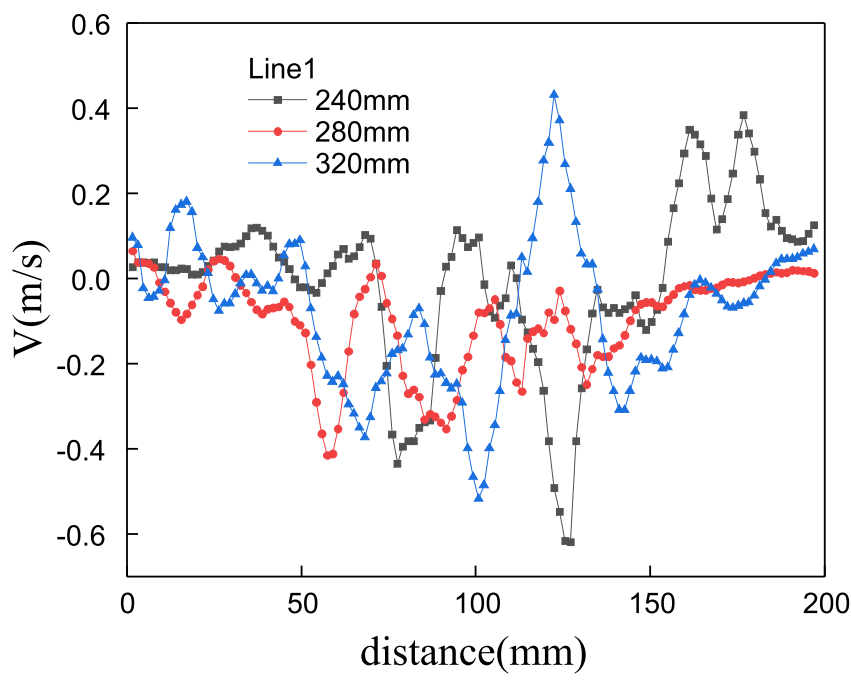

Figure 11.1. Axial velocity distribution of particles under different irradiation cross sections on line 1

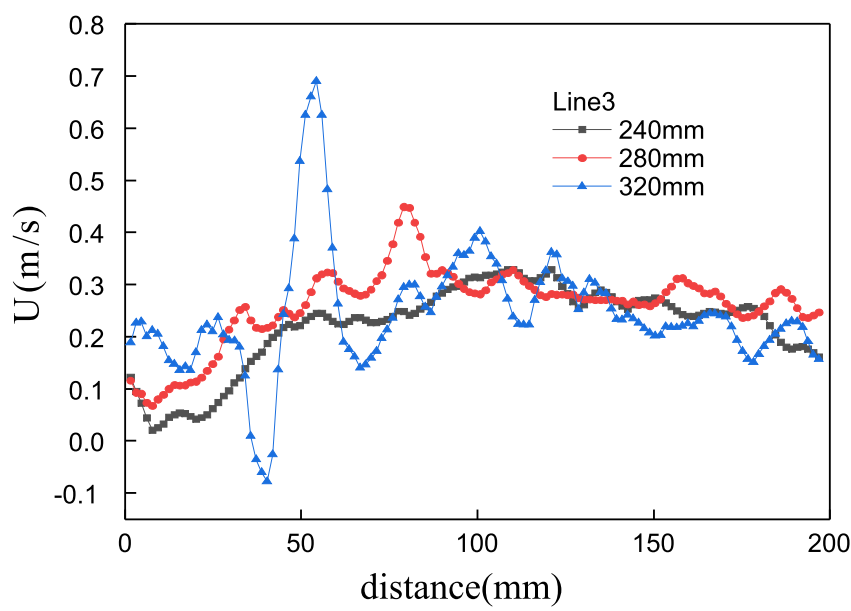

Figure 11.3. Axial velocity distribution of particles under different irradiation cross sections on line 3 has a weak impact on the axial velocity of particles at the edge of the tank wall.

Combined with Fig. 10.4 and Fig. 11.4, it can be clearly judged that the radial and axial particle velocity near the wall at different cross section heights shows a downward trend, but when $r>0.7 \mathrm{R}$, the particle velocity doesn't significantly with the decrease of section height, and the particle energy changes little, so it is considered that the damage in this area cannot be ignored. To sum up, it can be considered that when the radius $r$ of the conical surface at the lower cone section of the sandblast cylinder is less than $0.7 \mathrm{R}$, the particles will collide and cut the tank wall to a certain extent, and the wear and thinning of the conical surface will be more serious with the decrease of the height. When $\mathrm{r}>0.3 \mathrm{R}$, more attention should be paid to the wear and thinning of the cross section thickness of the sandblast cylinder.

Test results under different filling conditions of the particles (particle size: $24 \#, H=240 \mathrm{~mm}$ )

In order to further analyze the particle velocity characteristics in the sandblast cylinder after the particles are continuously ejected during the working process, the characteristics of the particles in the sandblast cylinder which move along with the change of sandblasting time are studied by taking the sandblasting time $t$ as a variable.

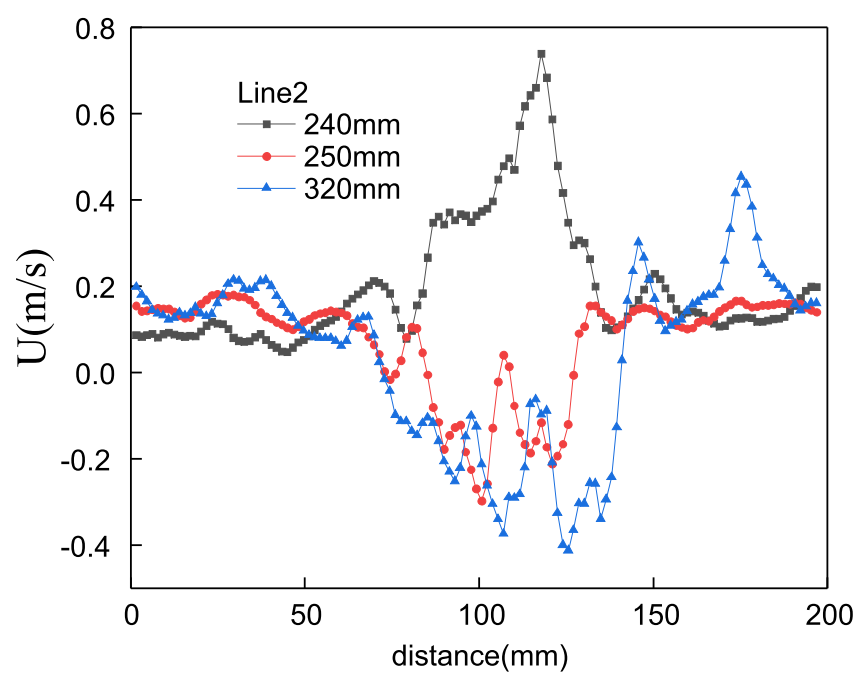

Figure 11.2. Axial velocity distribution of particles under different irradiation cross sections on line 2

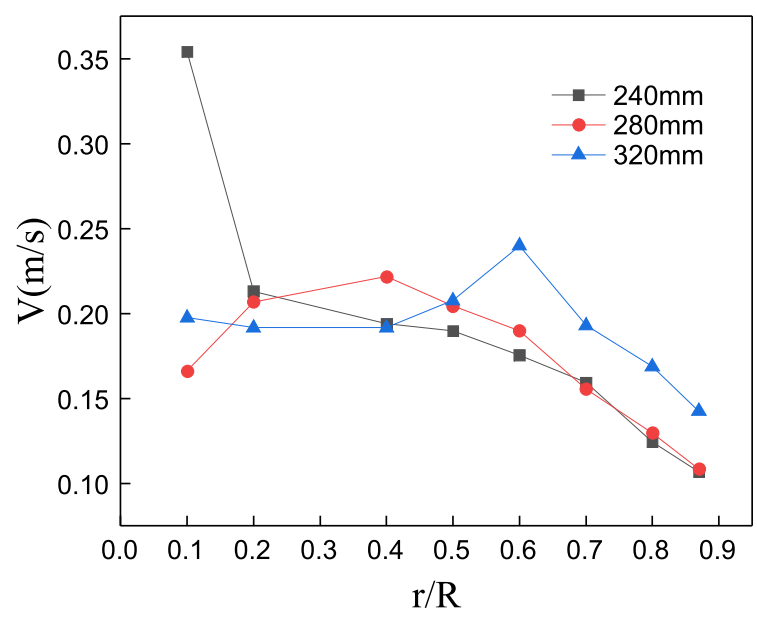

Figure 11.4. Axial velocity distribution of particles under different irradiation cross sections along different circular section trajectories 
It is defined that the sandblasting time $t<6 \mathrm{~s}$ is large number of particles filling state $\left(\mathrm{NUM}_{\mathrm{P} 1}\right)$, the sandblasting time $6 \mathrm{~s} \leq \mathrm{t} \leq 12 \mathrm{~s}$ is the medium particle number filling state $\left(\mathrm{NUM}_{\mathrm{P} 2}\right.$, and the sandblasting time $\mathrm{t}>12 \mathrm{~s}$ is the least particle number filling state $\left(\mathrm{NUM}_{\mathrm{P} 3}\right)$.

\section{Velocity vector distribution under different filling con- ditions of the particles}

As shown in Fig. 12.1 to Fig. 12.3, it can be seen that the particle velocity distribution is more uniform in the filling state of larger number of particles, and the particle velocity disturbance is more obvious in the filling state of less number of particles.

\section{Radial velocity vector $(\mathrm{U})$ distribution under different filling conditions of the particles}

As shown in Fig. 13.1 to Fig. 13.3, (1) the radial velocity of particles in different particle filling state along line 1 to line 3 presents a fluctuant distribution from the tank center to the both sides of the tank wall, and the peak (absolute value) is near the center $(50 \mathrm{~mm} \sim 150 \mathrm{~mm})$. The peak value appears on the right side of the tank center, and it gradually decreases with the time, and the radial velocity at the edge of the section is around 0 ; (2) On line 1, the radial velocity is larger under the filling state of less number of particles; on center line 2, the radial velocity is larger under the filling state of

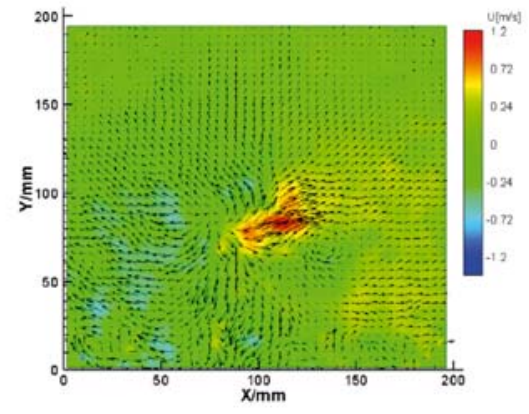

Figure 12.1. Velocity vector distribution under the condition of large number of particles

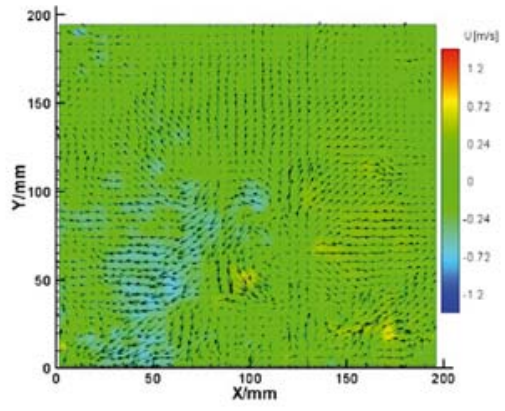

Figure 12.2. Velocity vector distribution under the condition of medium number of particles

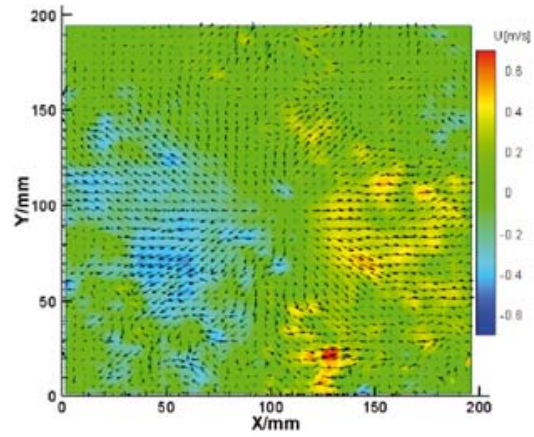

Figure 12.3. Velocity vector distribution under the condition of less number of particles

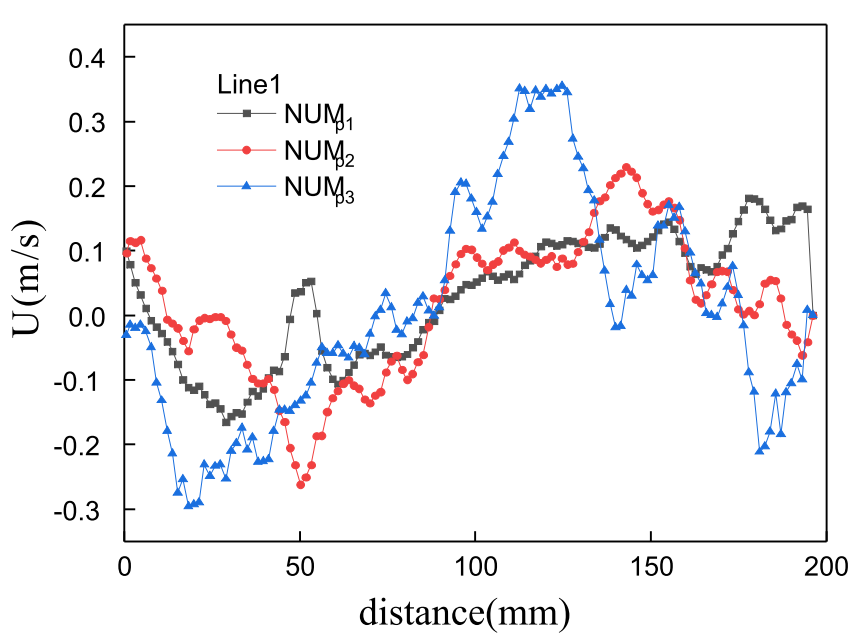

Figure 13.1. Radial velocity distribution of particles under different filling conditions of the particles on line 1

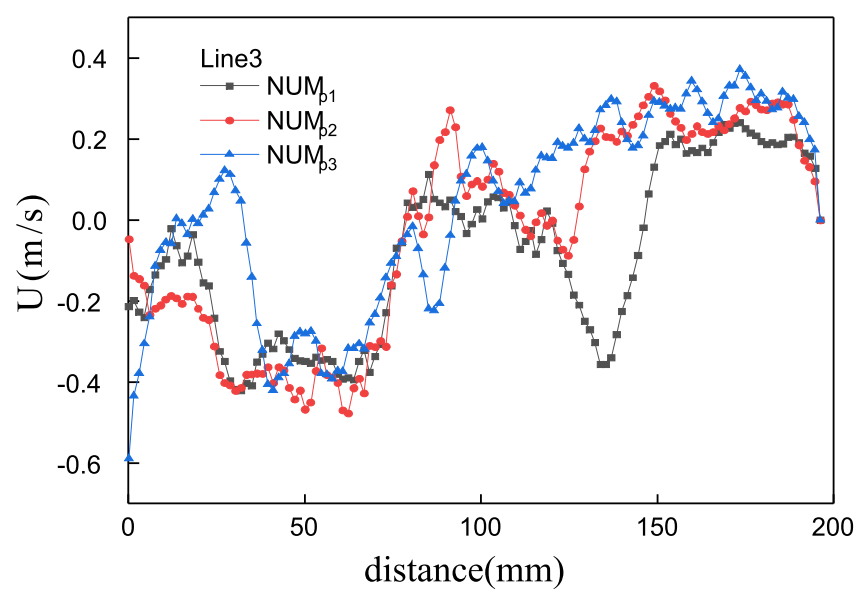

Figure 13.3. Radial velocity distribution of particles under different filling conditions of the particles on line 3

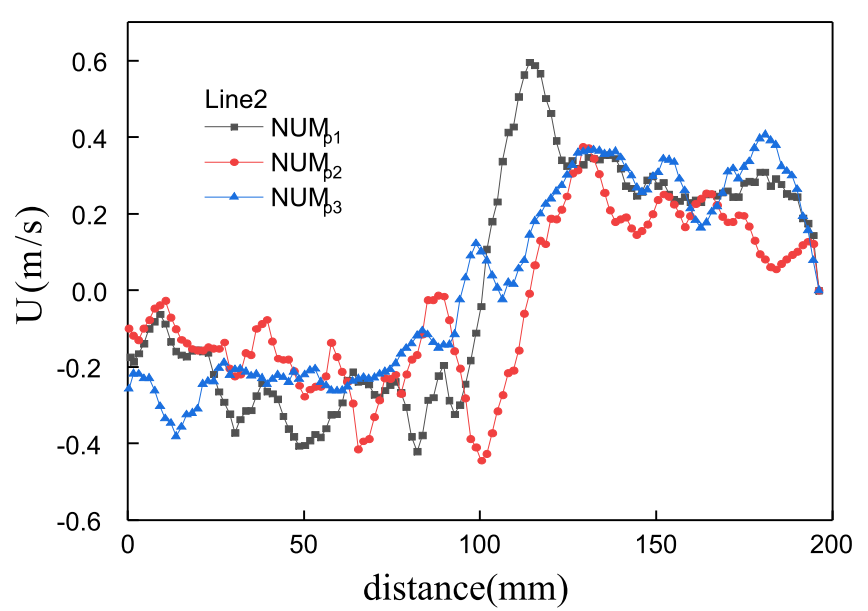

Figure 13.2. Radial velocity distribution of particles under different filling conditions of the particles on line 2

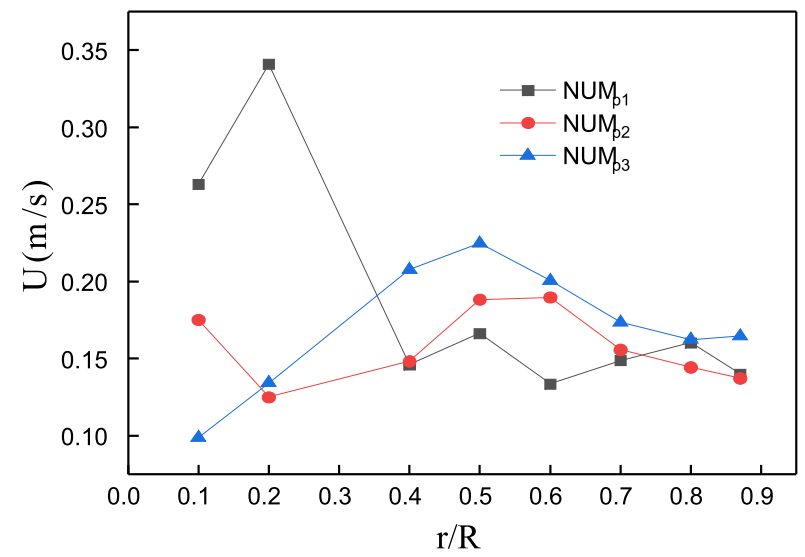

Figure 13.4. Radial velocity distribution of particles under different filling conditions of the particles along different circular section trajectories 
large number of particles; on line 3 , the particle filling state has no obvious influence on the maximum radial velocity of particles.

In Figure 13.3, Nump3 drops at the value of $140 \mathrm{~mm}$ accessories. This is because of the uneven distribution of gas and gas phases at both sides caused by the local accumulation of particles when they flowed out of the sandblasting tank, resulting in the local increase in the momentum exchange between gas and solid phases at one side, as well as the jump of particle velocity.

It can be seen from Fig. 13.4 that in the process that the particles move from the center to the tank wall in different particle filling states, the radial velocity shows a gradual downward trend, and the velocity value is relatively close. The radial velocity of particles near the wall in the filling state of large number of particles decreases more obviously, which indicates that the larger the particle handling capacity in the sandblast cylinder is, the smaller the radial particle velocity near the tank wall will be. The energy and quantity of a single particle are integrated to analyze the energy situation of the whole cross-section particles. To comprehensively measure the total energy produced by the number and speed of particles and its impact on the cylinder wall wear, parameter $\mathrm{S}$ is defined to characterize $S_{1}$ : results of energy integration in the filling state of large number of particles; $\mathrm{S}_{2}$ : results of energy integration in the filling state of medium number of particles; $\mathrm{S}_{3}$ : results of energy integration in the filling state of less number

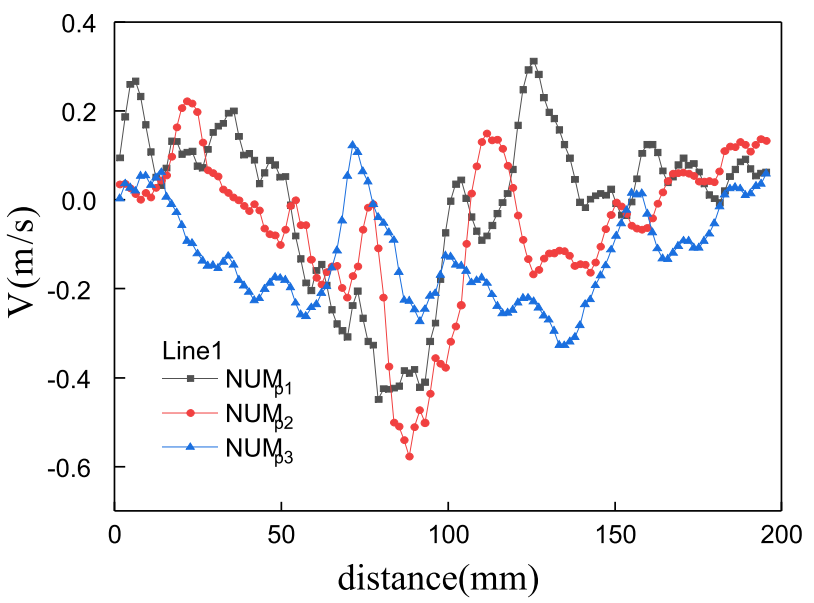

Figure 14.1. Axial velocity distribution of particles under different filling conditions of the particles on line 1

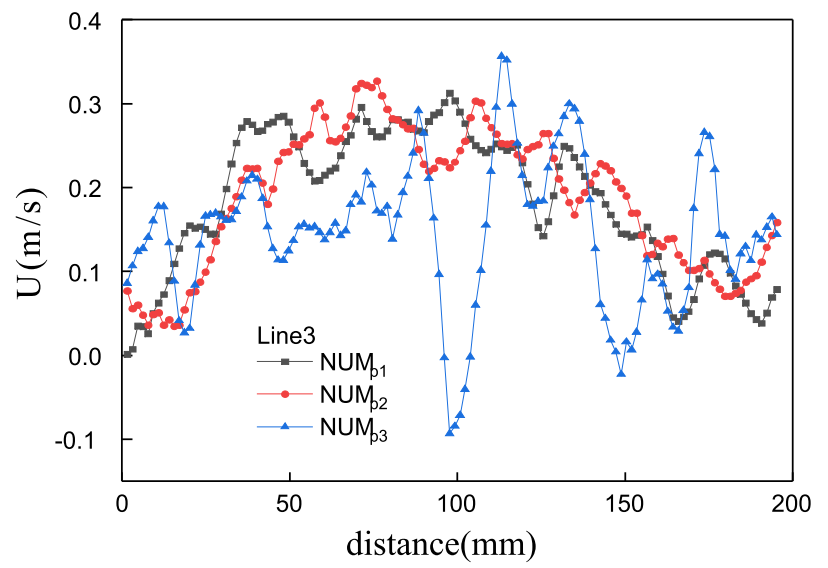

Figure 14.3. Axial velocity distribution of particles under different filling conditions of the particles on line 3 of particles. It is found that $S_{1}=0.150, S_{2}=0.120$, and $\mathrm{S}_{3}=0.137$. Although the radial velocity is small in the filling state of large number of particles, the number of particles is large, and the overall energy is high, thus resulting in a serious overall wear. Although the energy of a single particle is high in the filling state of less number of particles, the number of particles is small, the impact may be correspondingly weak. However, in the middle filling state, the number and speed of particles reach an equilibrium state, the overall energy value is the lowest $\left(S_{2}=0.120\right)$, and the wear of cylinder wall is the smallest.

\section{Axial velocity vector (V) distribution under different filling states of the particles}

As shown in Fig. 14.1 to Fig. 14.3, the axial velocity of particles in different particle filling states along line 1 to line 3 presents a fluctuant distribution from the tank's center to both sides of the tank wall, and it reaches the peak (absolute value) near the center. Then the velocity decreases gradually in the form of fluctuation, and the radial velocity at the edge of the section is all around 0 .

It can be seen from Fig. 14.4 that in the process that the particles move from the center to the tank wall in different particle filling states, the axial velocity tends to decrease gradually, and the axial velocity value is relatively close. In addition, the value is lower than the radial velocity value at the same position.

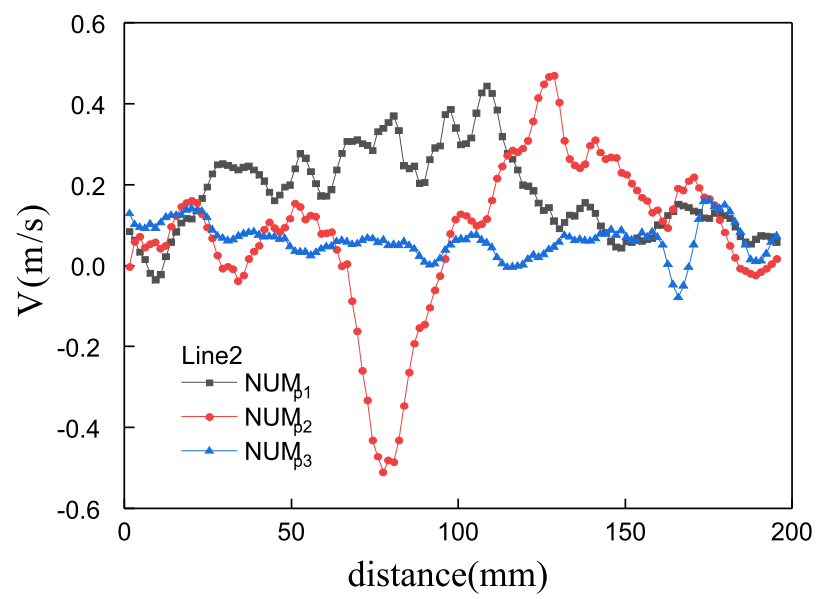

Figure 14.2. Axial velocity distribution of particles under different filling conditions of the particles on line 2

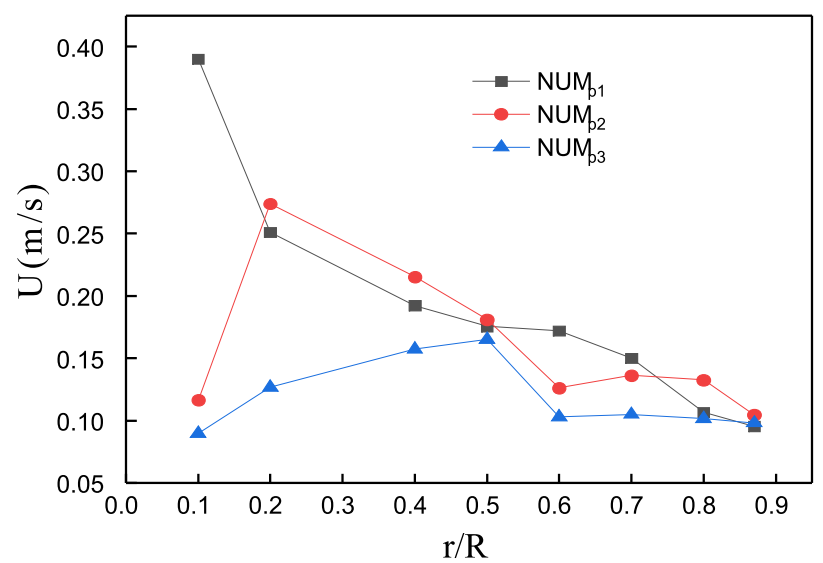

Figure 14.4. Axial velocity distribution of particles under different filling conditions of the particles along different circular section trajectories 


\section{CONCLUSIONS}

Influenced by the structural characteristics of sandblast cylinder model, gas inflow, particle outflow and particle diameter unevenness, the motion characteristics of the particles on the observed cross-section in the test are asymmetric, and the vortex and disturbance appear in some areas. However, these areas are mainly located in the center of the section, as shown in Fig. 7.4, such as the abnormal velocity of 24\# particles at $\mathrm{r} / \mathrm{R}=0.2$ shown in Fig. 7.1, the abnormal velocity of particles $30 \#$ at $x=110 \mathrm{~mm}$ and so on. A further study can be carried out to the abnormal movement of particles in these areas. In this paper, the movement trend of particles in the area away from the center and the possible wear on the tank wall were mainly studied, and it can be concluded that it doesn't affect the following conclusions.

(1) Influenced by the same other factors, the velocity distribution range of particles is gradually narrowed with the increase of particle diameter in the tank. However, the radial velocity of particles with a smaller particle diameter decreases more obviously, so the particles with a larger diameter have more energy when they approach the wall surface, thus producing a larger wear on the wall surface.

(2) Under the action of different particle diameter, crosssection height and particle filling state, the radial velocity and axial velocity of particles are reduced to a lower level when they are close to the cylindrical wall of the sandblast cylinder. However, the influence of relevant factors on the radial velocity of particles is greater than that on the axial velocity. The weakening degree of the particles' axial velocity is more obvious when they are close to the wall of the sandblast cylinder than the radial velocity. The radial impact wear of the particles on the wall of sandblast cylinder is greater than the axial cutting wear.

(3) Under the filling state of large number of particles, although the speed of a single particle is small, the number of particles is large. In the middle filling state, the number and speed of particles reach an equilibrium state, the overall energy value is the lowest, and the wear of cylinder wall is the smallest.

(4) Under the influence of different factors, the radial velocity and axial velocity of particles from the center to the tank show a downward trend. When $r>0.7 \mathrm{R}$, the velocity decreases obviously. When $r>0.3 \mathrm{R}$, the particles' velocity maintains a high level. Therefore, for the structural size of the sandblast cylinder of the test model, we should focus on the abrasion of the parts whose section radius of the conical section of the sandblast cylinder $r$ $<0.7 \mathrm{R}$, especially the abrasion of the particles on the tank wall at the parts with the section radius $r>0.3 R$.

\section{ACKNOWLEDGMENTS}

This work is financed by the science and technology project of Guangdong market supervision and Administration Bureau of China (grant no. 2018CT26), science and technology research project of Guangzhou (grant no. 202002030140).

\section{LITERATURE CITED}

1. Juanjuan,S.U., Lihua,L.V. \& Gaojin, L.I. et al. 2017. Research and Application of Continuous Sand-Feeding Sand Blaster[J]. Marine Technol. 65-67, 92.
2. Yilong, H.E., LIU, Sshuling \& HU, Jianxiu. 2019. Choose and Application of OCTG Internal Wall Blasting Machine[J]. Chem. Engin. \& Equipment, 2(02), 167-169. DOI: 10.19566/j. cnki.cn35-1285/tq.2019.02.071.

3. Ri Zhanga, Haixiao Liub \& Sheng Donga. 2019. Approximate theoretical solution of the movement and erosion of solid particles in a $90^{\circ}$ bend [J]. Wear. 430-431 (2019) 233-244. DOI: 10.1016/j.wear.2019.05.013.

4. Thon, A., Püttmann, A. \& Hartge, E.U. et al. 2011. Simulation of catalyst loss from an industrial fluidized bed reactor on the basis of labscale attrition tests[J]. Powder Technol. 214 (1), 21-30. .DOI: 10.1016/j.powtec.2011.07.017

5. Werther, J. \& Xi, W. 1993. Jet attrition of catalyst particles in gas fluidized beds[J]. Powder Technol. 76 (1), 39-46. DOI: 10.1016/0032-5910(93)80039-D.

6. Solnordal, C.B., Wong, C.Y. \& Boulanger, J., 2015. An experimental and numerical analysis of erosion caused by sand pneumatically conveyed through a standard pipe elbow, Wear 336 43-57. DOI: 10.1016/j.wear.2015.04.017.

7. Oka, Y.I., Okamura, K. \& Yoshida, T. (2005). Practical estimation of erosion damage caused by solid particle impact: part 1: effects of impact parameters on a predictive equation, Wear 259, 95-101.

8. Oka, Y. \& Yoshida, T. (2005). Practical estimation of erosion damage caused by solid particle impact: part 2: mechanical properties of materials directly associated with erosion damage, Wear, 259, 102-109.

9. Ballout, Y.,Mathis, J.A. \& Talia, J.E. (1996). Solid particle erosion mechanism in glass[J]. Wear, 196(1). DOI: 10.1016/00431648(96)06922-0.

10. Simonini, A., Theunissen, R., Masullo, A. \& Vetrano, M.R. (2019). PIV adaptive interrogation and sampling with image projection applied to water sloshing[J]. Experim. Thermal Fluid Sci. 102. DOI: 10.1016/j.ijheatfluidflow.2020.108561.

11. Israel González-Neria, Alejandro Alonzo-Garcia, Sergio, A., Martínez-Delgadillo, Víctor X. Mendoza-Escamilla, Juan Antonio Yáñez-Varela, Patrick G. Verdin \& Gabriela Rivadeneyra-Romero. (2019). PIV and dynamic LES of the turbulent stream and mixing induced by a V-grooved blade axial agitator[J]. Chem. Engin. J. 374. DOI: 10.1016/j.cej.2019.06.033.

12. Sunghyuk Im, Hyoung Tae Kim, Bo Wook Rhee \& Hyung Jin Sung. (2016). PIV measurements of the flow patterns in a CANDU-6 model[J]. Annals of Nuclear Energy, 98. DOI: 10.1016/j.anucene.2016.07.012.

13. Nguyen Lu Phuong \& Kazuhide Ito. (2015). Investigation of flow pattern in upper human airway including oral and nasal inhalation by PIV and CFD[J]. Buil. Environ., 94. DOI: 10.1016/j.buildenv.2015.10.002.

14. Jacobi, G., Thill, C.H., van't Veer, R. \& Huijsmans, R.H.M. (2019). Analysis of the influence of an interceptor on the transom flow of a fast ship by pressure reconstruction from stereoscopic scanning PIV[J]. Ocean Engin. 181. DOI: 10.1016/j.oceaneng.2019.02.062.

15. Sijie Fu, Pascal Henry Biwole \& Christian Mathis. (2016). Numerical and experimental comparison of 3D Particle Tracking Velocimetry (PTV) and Particle Image Velocimetry (PIV) accuracy for indoor airflow study[J]. Buil. Environ., 100. DOI: 10.1016/j.buildenv.2016.02.002.

16. Sijie Fu, Pascal Henry Biwole \& Christian Mathis. (2015). Particle Tracking Velocimetry for indoor airflow field: A review[J]. Buil. Environ., 87. DOI: 10.1016/j.buildenv.2015.01.014.

17. Feng Wu, Zeyu Yu, Lingyi Shang, Xiaoxun Ma \& Wenjing Zhou. (2019). Experimental investigation on hydrodynamic behavior in a spouted bed with longitudinal vortex generators[J]. Adv. Powder Technol. 30(10). DOI: 10.1016/j.apt.2019.06.033. 